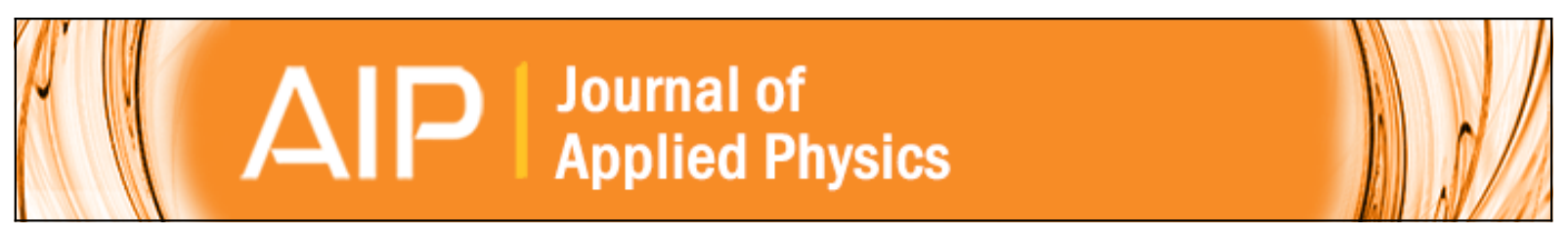

\title{
Photocapacitance study of type-II GaSb/GaAs quantum ring solar cells
}

M. C. Wagener, P. J. Carrington, J. R. Botha, and A. Krier

Citation: Journal of Applied Physics 115, 014302 (2014); doi: 10.1063/1.4861129

View online: http://dx.doi.org/10.1063/1.4861129

View Table of Contents: http://scitation.aip.org/content/aip/journal/jap/115/1?ver=pdfcov

Published by the AIP Publishing

\section{Articles you may be interested in}

Effect of Sb induced type II alignment on dynamical processes in InAs/GaAs/GaAsSb quantum dots: Implication to solar cell design

Appl. Phys. Lett. 103, 072112 (2013); 10.1063/1.4818762

Simulation of the enhanced infrared photoresponse of type-II GaSb/GaAs quantum ring solar cells

Appl. Phys. Lett. 103, 063902 (2013); 10.1063/1.4818126

Enhanced infrared photo-response from $\mathrm{GaSb} / \mathrm{GaAs}$ quantum ring solar cells

Appl. Phys. Lett. 101, 231101 (2012); 10.1063/1.4768942

Type-II GaSb/GaAs coupled quantum rings: Room-temperature luminescence enhancement and recombination lifetime elongation for device applications

Appl. Phys. Lett. 101, 031906 (2012); 10.1063/1.4737443

Thermal emission in type-II GaSb/GaAs quantum dots and prospects for intermediate band solar energy conversion

J. Appl. Phys. 111, 074514 (2012); 10.1063/1.3703467

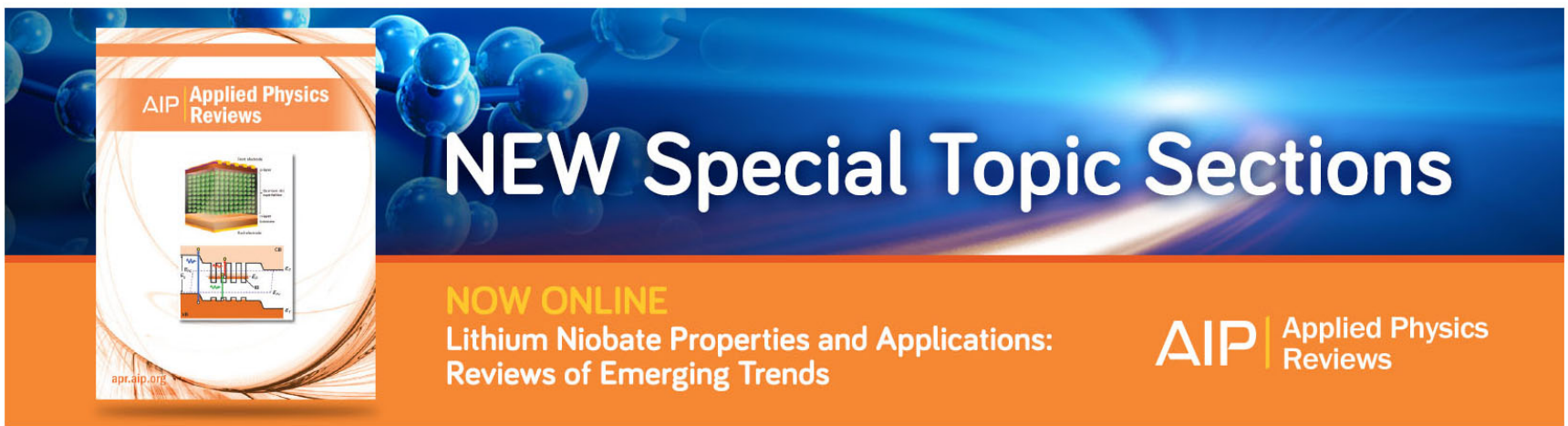




\title{
Photocapacitance study of type-II GaSb/GaAs quantum ring solar cells
}

\author{
M. C. Wagener, ${ }^{1}$ P. J. Carrington, ${ }^{2}$ J. R. Botha, ${ }^{1}$ and A. Krier $^{2}$ \\ ${ }^{1}$ Department of Physics, Nelson Mandela Metropolitan University, Port Elizabeth, South Africa \\ ${ }^{2}$ Department of Physics, Lancaster University, Lancaster LA1 4YB, United Kingdom
}

(Received 8 August 2013; accepted 16 December 2013; published online 6 January 2014)

\begin{abstract}
In this study, the density of states associated with the localization of holes in $\mathrm{GaSb} / \mathrm{GaAs}$ quantum rings are determined by the energy selective charging of the quantum ring distribution. The authors show, using conventional photocapacitance measurements, that the excess charge accumulated within the type-II nanostructures increases with increasing excitation energies for photon energies above $0.9 \mathrm{eV}$. Optical excitation between the localized hole states and the conduction band is therefore not limited to the $\Gamma(\mathrm{k}=0)$ point, with pseudo-monochromatic light charging all states lying within the photon energy selected. The energy distribution of the quantum ring states could consequently be accurately related from the excitation dependence of the integrated photocapacitance. The resulting band of localized hole states is shown to be well described by a narrow distribution centered $407 \mathrm{meV}$ above the GaAs valence band maximum. C 2014 AIP Publishing LLC. [http://dx.doi.org/10.1063/1.4861129]
\end{abstract}

\section{INTRODUCTION}

Low dimensional devices have been intensively studied as a means to improve the conversion efficiency of optical sensors. In particular, the reduced strain associated with quantum rings (QRs) has made it possible to incorporate multiple QR stacks into the junction of a $p-i-n$ solar cell without the formation of extended defects. ${ }^{1}$ The associated increase in the short-circuit current is predominantly related to the extended spectral response of the solar cell. In addition to the optical transitions from the GaSb QR to the GaAs conduction band continuum ( $\mathrm{QR}$ charging), optical hole emission ( $\mathrm{QR}$ discharging) is also anticipated. Depending on the distribution of the localized hole states within the band gap, the intermediate band nature of the QR states is therefore expected to further improve the conversion efficiency under concentrated excitation. This study therefore sets out to describe the optical charging of the QRs by monitoring the photocapacitance of the solar cell under various excitation conditions.

\section{EXPERIMENTAL DETAILS}

The solar cell structure used in this study was deposited using a VG-V80H molecular beam epitaxy reactor. A $3 \mu \mathrm{m}$ thick GaAs base layer was grown with a doping density of $10^{17} \mathrm{~cm}^{-3}$, followed by a $400 \mathrm{~nm}$ undoped region containing ten $\mathrm{GaSb}$ QR stacks separated by $40 \mathrm{~nm}$ GaAs spacers. The structure was completed with a $0.5 \mu \mathrm{m}$ thick p-type emitter and a capped $\mathrm{Al}_{0.8} \mathrm{Ga}_{0.2}$ As window layer. A detailed description of the growth procedure and the device structure is given in Ref. 1. Cross-sectional transmission electron microscopy images showed the rings to have a typical outer and inner diameter of $23 \mathrm{~nm}$ and $10 \mathrm{~nm}$, respectively, a thickness of approximately $1.7 \mathrm{~nm}$, and a typical surface density of $10^{10}$ rings $/ \mathrm{cm}^{2}{ }^{1}$ These structures were processed into circular solar cells with a diameter of $1 \mathrm{~mm}$ using standard photolithography. The photocapacitance was measured using a pseudo-monochromatic light source and a Boonton $7200 \mathrm{ca}-$ pacitance meter operating at a $1 \mathrm{MHz}$ modulation frequency.
The low temperature measurements were performed in a closed-cycle helium cryostat.

\section{RESULTS AND DISCUSSION}

Figure 1 compares the low temperature $(16 \mathrm{~K})$ photocapacitance-voltage $(C-V)$ curves obtained under different illumination conditions. Although the dark $C$ - $V$ curve is relatively parabolic, the $C-V$ curves obtained under illumination are characterized by two capacitance plateaus (labelled by $C_{\mathrm{I}}$ and $C_{\mathrm{II}}$ ). The two plateaus indicate two charge states, with $C_{\mathrm{II}}$ attributed to an increased charge accumulation within the QRs. Following the approach used by Wang et al., ${ }^{2}$ the difference in capacitance between the two charge states relates to an excess charge of $6 \times 10^{10}$ holes $/ \mathrm{cm}^{2}$ or almost one hole per quantum ring. The transition voltage from state $C_{\mathrm{II}}$ to $C_{\mathrm{I}}$ is also found to shift from $-4.5 \mathrm{~V}$ reverse bias for a photon energy of $1.55 \mathrm{eV}$ $(\lambda=800 \mathrm{~nm})$ up to $1 \mathrm{~V}$ forward bias for a photon energy of $885 \mathrm{meV}(\lambda=1400 \mathrm{~nm})$. The photocapacitance associated with the accumulated charge within the QRs is therefore clearly

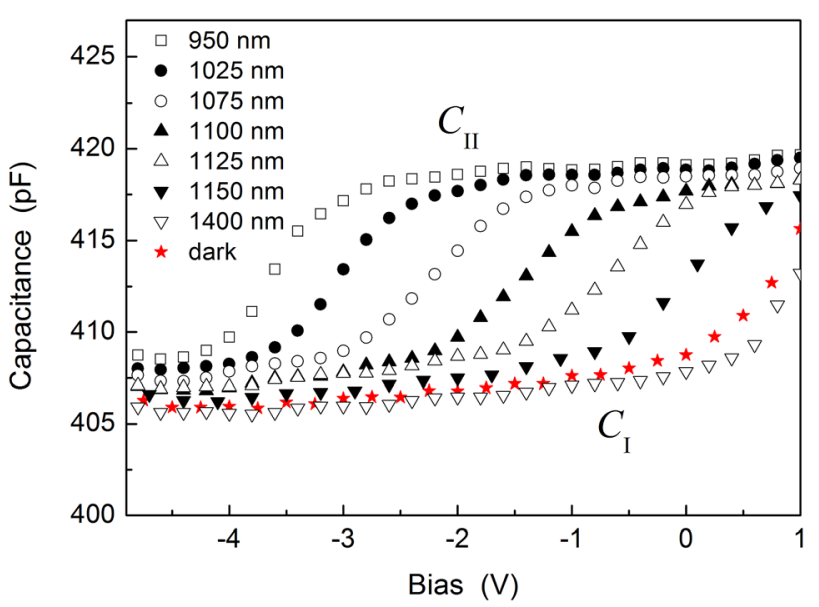

FIG. 1. Low temperature (16 K) capacitance-voltage curves of a GaSb/GaAs solar cell under various excitation wavelengths. 
controlled by the photon energy, increasing steadily for excitation energies above approximately $0.9 \mathrm{eV}$. It should also be pointed out that the $C-V$ curves were independent of the sequence in which they were measured. From this, we concluded that the thermal emission rate was sufficient to return the QRs to equilibrium, following excitation. Furthermore, the $C$ - $V$ curve measured using $1400 \mathrm{~nm}$ excitation was found to lie below the $C-V$ curve measured in the dark. A slight decrease (discharging) of the QR charge was therefore observed when using sufficiently low excitation energies. The charge density per unit area in a quantum well has previously been described by Wang et al., ${ }^{2}$ with the excess charge given by

$$
Q=\int_{V}\left(C-C_{I}\right) d V
$$

where $C$ represents the measured photocapacitance and $V$ is the applied bias. It is therefore clear that illuminating with photon energies near the absorption edge (open squares in Fig. 1) produces the greatest accumulation of charge within the space-charge region, with lower energies producing a systematic decrease in excess charge. Illuminating with a pseudo-monochromatic light source with energy, $E_{\mathrm{P}}$, therefore results in the charging of all states within the excitation range of the illumination. The optical transitions are therefore not limited to the $\Gamma(k=0)$ point (as also deduced from a photo-response study described in Ref. 3), with the net charge described in terms of the integrated QR hole state density, $N(\mathrm{E})$, that is

$$
Q\left(E_{p}\right)=e \int_{0}^{E p} N(E) d E
$$

By performing a series of photocapacitance measurements, it would then be possible to obtain the density of states (DOS) at $E_{\mathrm{p}}$ using the relation

$$
N\left(E_{P}\right)=\frac{1}{e} \frac{\partial Q\left(E_{p}\right)}{\partial E_{p}}=\frac{1}{e} \frac{\partial}{\partial E_{p}}\left[\int_{V} C d V\right]_{E_{p}} .
$$

In other words, in order to determine the density of states from the photocapacitance-voltage profiles, the integrated photocapacitance is required for a number of closely spaced excitation energies, with the accuracy of $N(E)$ limited by the differentiation operation. Figure 2 gives the energy dependence of the integrated photocapacitance depicted in Fig. 1. The systematic increase in the localized hole charge within the $p-i-n$ diode is characterized by a sharp increase at roughly $h \nu=1.1 \mathrm{eV}$ and a second step at $h \nu=1.35 \mathrm{eV}$. The localized hole states associated with the increased spacecharge are therefore concentrated around these particular photon energies, with both contributions saturating for excitation energies near the GaAs band gap energy.

Also indicated is the integrated capacitance without optical excitation (dashed line). Since the capacitance measurements were performed at $16 \mathrm{~K}$, the thermal emission rate was

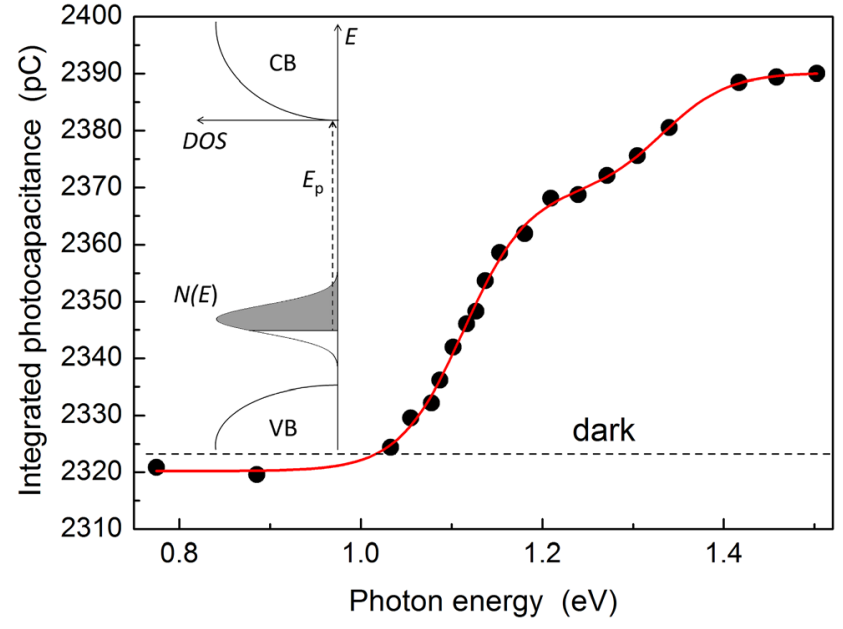

FIG. 2. Energy dependence of the integrated photocapacitance measured under different excitation conditions. The solid line represents the best fit using a double Boltzmann function. The integrated capacitance obtained without excitation is represented by the dashed line. The inset illustrates the band segment of $N(E)$ charged (area in grey) with optical excitation $h \nu=E_{\mathrm{p}}$.

sufficiently low (relative to the photon flux) for the QR charge to become saturated. However, since the integrated photocapacitance decreases below the dark value for small photon energies $(h \nu<0.9 \mathrm{eV})$, there also appears to be a certain degree of optical emission (optical discharging) of holes from the quantum ring states. Although a relatively small effect, optical discharging was observed for a variety of $\mathrm{GaSb} / \mathrm{GaAs}$ solar cells, only dissipating when thermal emission became dominant (temperatures above $150 \mathrm{~K}$ ).

In order to estimate the density of states from the integrated photocapacitance, a double Boltzmann function (Scurve) was fitted to the measured values and differentiated in terms of the photon energy. This is essentially equivalent to describing the quantum ring states by two Gaussian distributions. Figure 3 shows the distribution of hole states, following differentiation. The DOS is represented by two bands with maxima $188 \mathrm{meV}$ and $407 \mathrm{meV}$ above the valence band maximum (VBM), where we have instead plotted the photon

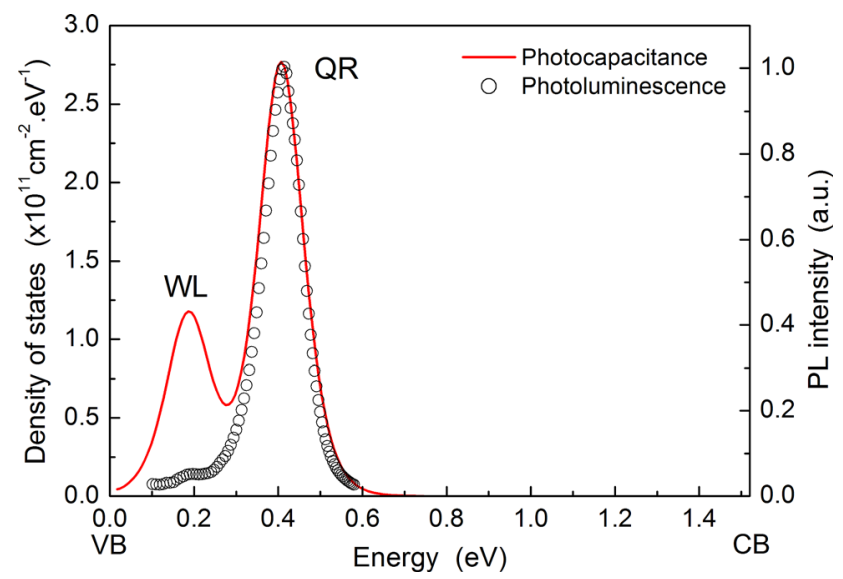

FIG. 3. Density of states estimated from the photon energy dependence of the integrated photocapacitance in Fig. 2 (solid line). The open circles represent the normalized hole energy distribution estimated from the $4.2 \mathrm{~K} \mathrm{PL}$ spectrum (i.e., band gap energy minus transition energy) of the same structure. 
energy, $E_{\mathrm{p}}$ relative to the GaAs band gap energy. Since the hole localization within the QR is expected to result in a single, discrete state due to the small effective mass of $\mathrm{GaSb} / \mathrm{GaAs}$ nanostructures, ${ }^{4}$ we have ascribed the finite width of the DOS to the inhomogeneous size distribution of the nanostructures. Using the approach described in Ref. 3, the band-width of the DOS could be related to a particular QR size distribution, leading to a mean QR height of $1.75 \mathrm{~nm}$ and a standard deviation of $0.25 \mathrm{~nm}$. Also given is the distribution of hole states estimated from the $4.2 \mathrm{~K}$ photoluminescence (PL) of the same structure. The PL spectrum, which was obtained under high excitation conditions, demonstrates a remarkable correlation with the photocapacitance analysis, with the PL associated with the $\mathrm{GaSb} / \mathrm{GaAs}$ quantum rings nearly perfectly matched to the deeper band of states. Since the secondary PL peak near $200 \mathrm{meV}$ is usually ascribed to the wetting layer (WL) formed during antimony deposition, we have tentatively assigned the second band of states to the two-dimensional confinement within the wetting-layer.

In a separate study, the energy distribution of the localized hole states was related to the extended photo-response associated with the $\mathrm{GaSb} / \mathrm{GaAs}$ quantum rings. ${ }^{3}$ By including optical transitions beyond the $\Gamma$-point, the authors showed that the band of QR hole states has a maximum density of states roughly $390 \mathrm{meV}$ above the VBM. Furthermore, due to the observed nature of absorption in the type-II GaSb/GaAs QRs, the simulated band of hole states could only accurately describe the high energy (increased hole localization) side of the distribution. The photocapacitance measurements therefore complement our previous analysis by accurately quantifying the complete energy distribution of the QR states.

Previous capacitance studies extracted the hole localization energies by monitoring the thermal emission rate from the $\mathrm{GaSb} / \mathrm{GaAs}$ quantum dots. By performing optical deeplevel transient spectroscopy (DLTS) of quantum dots embedded in a GaAs $\mathrm{n}^{+}$-p junction, Geller et al. ${ }^{5,6}$ showed that the thermal activation energy increased from roughly $250 \mathrm{meV}$ to $450 \mathrm{meV}$ with decreasing excitation energy, with the optical DLTS signal quenching completely for excitation energies below $0.98 \mathrm{eV}$. Although our results correlate very well with the energy-selective charging observed by Geller et al., there remains a subtle difference in the interpretation. In this work, we show that charging occurs across an increasing segment of a single band of QR states, whereas the charging of sub-ensembles of dots/rings was previously proposed. The resonant optical charging of a specific charge state (as would be required for optical-multiplexing ${ }^{5}$ ) therefore seems to be impractical. Furthermore, the reported decrease in the thermal activation energy with decreasing reverse $\operatorname{bias}^{6-8}$ is unlikely to be attributed to state-filling, but instead describes the range of localization energies stemming from the size distribution of the $\mathrm{GaSb} / \mathrm{GaAs}$ quantum structures. In fact, in another DLTS study by Magno et al. ${ }^{9}$ the energy distribution was estimated by relating the DLTS peak height to its corresponding activation energy. The result was a distribution that extended up to $550 \mathrm{meV}$ with a maximum near $400 \mathrm{meV}$. From a comparison of the DLTS reports and our photocapacitance results, we therefore propose that the upper activation energy observed by DLTS does not represent the hole ground state, but instead the maximum localization energy represented within the band of hole states.

In summary, we have determined the energy distribution of holes localized within $\mathrm{GaSb} / \mathrm{GaAs}$ quantum rings using photocapacitance measurements. In the case of a type-II band structure, we find that optical absorption involves the integrated segment of hole states, lying within the excitation energy used. With this description of the charging process, we were able to show that the inhomogeneous quantum ring characteristics gives rise to a narrow band of hole states, $407 \mathrm{meV}$ above the GaAs valence band maximum.

\section{ACKNOWLEDGMENTS}

Financial support for this work was provided from EPSRC (Grant No. EP/G070334/1), as well as by the South African Research Chairs Initiative of the Department of Science and Technology, South African National Research Foundation and NMMU.

${ }^{1}$ P. J. Carrington, M. C. Wagener, J. R. Botha, A. M. Sanchez, and A. Krier, Appl. Phys. Lett. 101, 231101 (2012).

${ }^{2}$ J. B. Wang, F. Lu, S. K. Zhang, B. Zhang, D. W. Gong, H. H. Sun, and X. Wang, Phys. Rev. B 54, 7979 (1996).

${ }^{3}$ M. C. Wagener, P. J. Carrington, J. R. Botha, and A. Krier, Appl. Phys. Lett. 103, 063902 (2013).

${ }^{4}$ C. E. Pryor and M.-E. Pistol, Phys. Rev. B 72, 205311 (2005).

${ }^{5}$ M. Geller, C. Kapteyn, E. Stock, L. Müller-Kirsch, R. Heitz, and D. Bimberg, Physica E 21, 474 (2004).

${ }^{6}$ M. Geller, C. Kapteyn, L. Müller-Kirsch, R. Heitz, and D. Bimberg, Appl. Phys. Lett. 82, 2706 (2003).

${ }^{7}$ J. Hwang, A. J. Martin, J. M. Millunchick, and J. D. Phillips, J. Appl. Phys. 111, 074514 (2012).

${ }^{8}$ T. Nowozin, L. Bonato, A. Högner, A. Wiengarten, D. Bimberg, W.-H. Lin, S.-Y. Lin, C. J. Reyner, B. L. Liang, and D. L. Huffaker, Appl. Phys. Lett. 102, 052115 (2013).

${ }^{9}$ R. Magno, B. R. Bennett, and E. R. Glaser, J. Appl. Phys. 88, 5843 (2000). 